\title{
Otolaryngology Practice in Covid 19 Era: A Road-Map to Safe Endoscopies
}

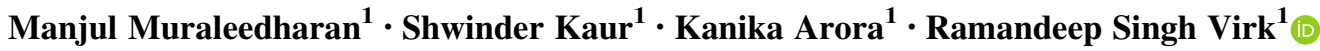

Received: 17 July 2020/ Accepted: 27 July 2020/Published online: 3 August 2020

(C) Association of Otolaryngologists of India 2020

\begin{abstract}
Covid-19 has changed the way medical services are being delivered all around the world. Otolaryngology as a speciality is anecdotally associated with high risk of infection. Endoscopies can be associated with aerosolization of particles due to cough or sneeze which may be induced. An overhaul of endoscopy and associated procedures is necessary keeping in mind the prevailing situations. This paper aims at a review of the on-going research and development of a road map for safe endoscopies-both for patients and heath care workers.
\end{abstract}

Keywords Otolaryngology · Endoscopy $\cdot$ Covid 19 . Safety

\section{Introduction}

Covid 19 or The Novel Corona Virus disease 2019 has changed the world as we know it forever. From the echelons of power to the humble dwellings, not a moment passes by without being concerned about the virus.

Endoscopies have been the game changers as far as the field of Otolaryngology is concerned. They have made the hitherto hidden areas of ear, nose and throat extremely accessible that, a practice without them, would be unthinkable and perceived grossly inadequate by the current crop of Otolaryngologists.

Ramandeep Singh Virk

virkdoc@hotmail.com

1 Department of Otolaryngology Head and Neck Surgery, Postgraduate Institute of Medical Education and Research (PGIMER), Chandigarh 160012, India
ENT Head and Neck Surgeons due to their proximity with the airway mucosa have been shown to be under increased risk of Covid 19 transmission. Endoscopies enhance this risk. Even though endoscopies per se aren't considered as Aerosol Generating Procedures (AGPs), they potentially increase the chances of cough, gag, and sneeze with subsequent aerosolization [1].The dangers are augmented by the fact that the Health Care Workers are in close proximity of the patient during the procedure. Virus particles which are aerosolized from mucosa may stay in air for $3 \mathrm{~h}$ of longer [2].

An analysis of the evidence available at present and framing a road map for safe diagnostic endoscopies in the Out Patient Practice are, thus, the need of the hour.

\section{Indications and Alternatives for Endoscopy}

Otolaryngology as a field has been found suitable for teleconsultation practices. Gilani et al. have reported that upto $80 \%$ of patients with ear complaints who underwent telemedicine consultation never required in person follow up visits [3]. According to $\mathrm{McCool}$ et al. too, patients with ear complaints were more suited for tele-consultation [4]. Soldatova et al. have described in detail a comprehensive approach for dysphagia evaluation designed for the present times [5]. It includes virtual history taking, assessment via virtual swallow evaluation and use of patient or care taker administered questionnaires and virtual therapy sessions. This reduces the in-patient visits and also brings down the necessity for invasive diagnostic procedures to the minimum.

As per the nasal endoscopy protocols put forth by Fried et al. only emergent/urgent cases should be considered for endoscopy-this includes tumor cases and refractory 
epistaxis [6]. They also suggest considering imaging modalities as the first line instead of endoscopies.

Setzen et al. also suggest revisiting CT as a substitute for nasal endoscopy and are in favour of getting a CT scan done, if facilities are available, thus negating the need for an endoscopy [2]. Obvious indications for nasal endoscopy include-unilateral symptoms, high suspicion of malignancy, failed appropriate medical treatment and complications of rhino sinusitis.

\section{Selection of Patients for Endoscopies}

The most important part is to screen the patients and identify the emergent/urgent cases out of the lot. Telemedicine facilities can be utilized for this. Imaging modalities are to be used when and where applicable.

Once the patient has been advised an endoscopy, an appointment should be given. This has to be arranged according to the available waiting space while ensuring proper social distancing. The number of care takers in the waiting area should be kept to the minimum number possible. The patients and attenders should be counselled regarding the higher than usual risk of transmission in the area given the presence of patients with upper respiratory symptoms, tracheostomy etc. A separate consent may be taken in this regard as per the existing local situation.

\section{Screening of the Patients and Attendants at the Entrance of Endoscopy Facility}

Thermal screening is necessary for all the patients and attenders.

The following questionnaire is to be filled by the screening physician so as to identify the high risk patients.

Travel History

History of travel to:

If yes, date of travel:

Symptoms

Fever: Yes/No date of onset:

Cough: Yes/No date of onset:

Sore throat: Yes/No date of onset:

Diarrhoea: Yes/No date of onset:

Respiratory distress: Yes/No date of onset:

Vomiting: Yes/No date of onset:

Clinical Impression: Covid suspicion Yes/No

If the patient or accompanying care-taker is of high suspicion for Covid infection, then quarantine and testing for the same are done as per the local infection control protocols.

\section{In the Waiting Area}

Care takers should be allowed only with seriously ill patients. Everyone in the waiting area should be wearing three plied surgical masks, properly. It should be mandatory to sanitize the hands with alcohol based hand rubs with greater than $60 \%$ ethanol or $70 \%$ isopropanol [7].

Patients with tracheostomy should be advised to wear a Heat Moisture Exchanger so as to prevent dissemination of aerosols generated during cough. In case of high risk cases surgical mask or N95 masks to be tied around the tube [8].

Appropriate social distancing is to be maintained in the waiting area. This might necessitate blocking off or removing furniture and moving patients along designated pathways [1].

\section{For Health Care Workers}

All the staff involved with the endoscopy room should undergo regular temperature check. Other precautions like regular hand hygiene, proper and regular use of masks should also be adhered to.

\section{Considerations During Endoscopy $[1,8]$}

The endoscopy room should have the bare minimum personnel present to avoid unnecessary exposure. The room should be cleared of unnecessary furniture, stationary and instruments. In cases where instruments or installations which are difficult to move are present care should be taken to cover them with water proof drapes.

The staff involved in the procedure should utilize PPEs-including masks (3 layered FFPs or N95s), cap, gown, shoe covers and gloves with proper donning and doffing.

A video system instead of direct visualisation through endoscope is preferred to keep the examiner as far from the patient as possible. It is also suggested that the endoscopy is recorded so as to avoid repeat procedures in case review is needed.

Mouth of the patient should be covered with three ply masks, if possible.

Endoscope tip and surrounding areas should be well lubricated with Xylocaine jelly. In case of preparation of nose for nasal endoscopy, nasal pledgets are to be used instead of aerosolised sprays. This is based on the fact that virus is harboured in nasopharynx [2]. [6] Similarly in case 90 degree rigid endoscopy for larynx, use of lignocaine viscous $10 \%$ should be preferred over $10 \%$ spray which usually is the practice at our institute. 
Suction tubing should be changed after every procedure and in-line filtration is preferred if feasible.

The endoscopic tables should be covered with disposable drapes and should be discarded after the procedure. The room should be cleaned meticulously after each procedure, which includes cleaning of all high touch and horizontal surface in procedure with $1 \%$ hypochlorite solution. Staff included in the cleaning of endoscopy rooms should utilize PPEs.

If the institutional policy and the economic considerations allow, it is preferable to use disposable endoscopic sheath for every patient.

Multiple innovations have also been introduced so as to reduce the direct exposure to aerosols during endoscopies. Maio et al. have described a back approach to patient where the operator positions herself/himself behind the patient and a flexible nasopharyngolaryngoscope is used. They also suggest avoiding all endoscopic procedures via oral cavity, nose being the safest route for endoscopic evaluation [9]. Thus flexible scopes are to be preferred to rigid ones for assessment of pharynx and larynx. This also helps in keeping the patients' facial mask on, reducing the spread via induced cough.

\section{Disinfection of the Scopes}

The Ministry of Health and Family Welfare, Government of India suggests sterilisation by immersing the scope in $2 \%$ glutaraldehyde solution for $20 \mathrm{~min}$ [8].

As per the Centre for Disease Control, $\geq 2.5 \%$ Glutaraldehyde $\left(\operatorname{Cidex}^{\mathrm{TM}}\right)$ for $5 \mathrm{~min}$ at $35^{\circ} \mathrm{C}$ can be used to disinfect scopes [10].

After disinfection, the endoscopes should be rinsed and channels flushed with sterile water/ filtered water/ or tap water to prevent adverse effects on patients associated with disinfectant retained on the endoscope.

\section{Conclusion}

The medical and surgical practices as we know now are in for an overhaul in the post pandemic period and Otolaryngology practice is no exception. The procedures and precautions mentioned above are based on the best possible evidence at present and hopefully would help in decreasing the incidences of heath care worker infection and cross infections.

Funding None.

Compliance with Ethical Standards

Conflict of interest None.

\section{References}

1. Iii JCD, Dubin MG, Ishman SL, Kuppersmith RB, Smith RV (2020) Guidance for return to practice for otolaryngology-head and neck surgery part one. https://www.entnet.org/sites/default/ files/uploads/guidance_for_return_to_practice_part_one_update_ 070120.pdf

2. Setzen M, Svider PF, Pollock K (2020) COVID-19 and rhinology: a look at the future. Am J Otolaryngol 15:102491

3. Gilani S, Bommakanti K, Friedman L (2020) Electronic consults in otolaryngology: a pilot study to evaluate the use, content, and outcomes in an academic health system. Ann Otol Rhinol Laryngol 129:170-174

4. McCool R, Davies L (2018) Where does telemedicine fit into otolaryngology? An assessment of telemedicine eligibility among otolaryngology diagnoses. Otolaryngol Head Neck Surg 158(4): 641-644

5. Soldatova L, Williams C, Postma GN, Falk GW, Mirza N (2020) Virtual dysphagia evaluation: practical guidelines for dysphagia management in the context of the COVID-19 pandemic. Otolaryngol Head Neck Surg 26:0194599820931791

6. Fried J, Imam SA, Lee JA, Nguyen SA (2020) Nasal endoscopy protocols in the era of COVID-19. World J Otorhinolaryngol Head Neck Surg. https://doi.org/10.1016/j.wjorl.2020.04.007

7. Healthcare Infection Control Practices Advisory Committee. Core infection prevention and control practices for safe healthcare delivery in all settings: recommendations of the healthcare infection control practices advisory committee (HICPAC) 2017

8. Mohfw.gov.in (2020) https://www.mohfw.gov.in/pdf/ENT COVID0306.pdf [cited 7 July 2020]

9. Di Maio P, Traverso D, Iocca O, De Virgilio A, Spriano G, Giudice M (2020) Endoscopic nasopharyngoscopy and ENT specialist safety in the COVID 19 era: the back endoscopy approach to the patient. Eur Arch Otorhinolaryngol 7:1

10. Rutala WA, Weber DJ (2020) Cdc.gov. 2020. https://www. cdc.gov/infectioncontrol/pdf/guidelines/disinfectionguidelines-H. pdf

Publisher's Note Springer Nature remains neutral with regard to jurisdictional claims in published maps and institutional affiliations. 